\title{
DESARROLLOS HIPERMEDIALES \\ DEL GRUPO LINGUATICs
}

Henry González
Esperanza Vera*

\begin{abstract}
This paper aims at socializing the two research projects proposed by the group LINGUATICs from Universidad Pedagógica Nacional, made up by 10 teachers from different content areas and 3 students, all belonging to the Language Department. The project "Hypermedia for the Development of Literature Didactics based On the short; short story HIMINI" seeks to integrate two apparently dissimilar disciplines, such as information Technology and Literature, in order to develop a new proposal regarding Literature Didactics; it is based on the most representative verbal literary work nowadays, that is, the short, short story The project "Design and Validation of a Hypermedia for the English Learning", seeks lo produce an interactive hypermedia game which can be sensitive to the difficulties the user can find when using it, so as lo pro vide him with information or training on effective strategies lo process information heard and interact with the characters, based on it.
\end{abstract}

\section{RESUMEN}

El presente artículo busca socializar los dos proyectos de investigación que hasta el momento ha generado el grupo LINGUATICs de la Universidad Pedagógica Nacional, conformado actualmente por 10 profesores de diferentes áreas y 3 estudiantes del Departamento de Lenguas: El proyecto "Ambiente Hipermedial para el Desarrollo de la Didáctica Literaria a partir del Minicuento, HIMINI", que busca integrar dos disciplinas aparentemente disímiles, como la informática y la literatura, para desarrollar una nueva propuesta de la didáctica literaria, recurriendo a la creación verbal más representativa del momento: el minicuento o minificción y, el proyecto "Diseño y Validación de un Ambiente Hipermedial para el aprendizaje del inglés" que se propone crear un juego interactivo que pueda ser sensible a las dificultades del usuario para proporcionarle información o entrenamiento en estrategias efectivas para procesar información verbal escuchada e interactuar con los personajes, con base en ella.

Palabras claves: Ambiente hipermedial, minicuento, didáctica de la literatura, Minificción Interactiva, portal, aprendizaje del inglés, estrategias de aprendizaje, comprensión auditiva en inglés, juego interactivo

\section{DESARROLLOS HIPERMEDIALES DEL GRUPO LINGUATICs}

El grupo Linguatics nació en el año 2001, en el marco del proyecto Ambientes computarizados para el aprendizaje de las Ciencias Humanas, en la Facultad de Humanidades de la Universidad Pedagógica Nacional, con el propósito de generar y realizar proyectos de Investigación e innovación, que den cuenta de la aplicación de las

\footnotetext{
*Profesores de tiempo completo e investigadores de la U.P.N.
} 
tecnologías informáticas y de las comunicaciones a los procesos de aprendizaje propios de las áreas del lenguaje, principalmente, la lingüística, la literatura y las lenguas extranjeras.

En su breve desarrollo el grupo Linguatics ha generado dos proyectos, que presentamos a continuación:

\section{Ambiente hipermedial para el desarrollo de la didáctica literaria a partir del minicuento. Himini.}

Los receptores literarios de nuestros días, especialmente aquellos que esperan obtener provecho pasando una parte de su vida en la institución escolar, expresan cada vez con mayor énfasis, la necesidad de que tanto las obras literarias como sus procesos de comprensión, estén más en concordancia con la dinámica de su tiempo. Es decir, esperan encontrar ambientes agradables de aprendizaje en los que tanto los textos como los maestros estén dotados de discursos leves, (menos acartonados, solemnes e impositivos) amenos, humorísticos y placenteros que les permita disfrutar el aprendizaje sin que ello se convierta en un sufrimiento o en la causa de múltiples penalidades.

En consonancia con los desafíos del aprendizaje en la escuela, también el desarrollo cultural de nuestro tiempo expresa sus propios retos a quienes desean interactuar con él. Uno de ellos, quizás el más Influyente, es el predominio de una cultura hegemonizada por el imperio de la imagen", en la que prácticas como el spot publicitario, el siegan político, el flash informativo, la historieta, el juego interactivo, el vídeo juego o el video clip le imprimen liviandad, rapidez, aprendizaje lúdico y dnamismo $A$ su vez, el signo distintivo de estas prácticas es la brevedad, principio que las relaciona con una de las creaciones estéticas verbales más distintivas de nuestra época como lo es el mini-cuento.

Teniendo en cuenta aquellas exigencias de la institución escolar, la dinámica adquirida por los discursos culturales de nuestra época y el impresionante desarrollo de las tecnologías de la comunicación, dos profesores de diferentes áreas, pertenecientes a la Facultad de Humanidades, decidieron proponer un proyecto de investigación en el que fuera posible integrar dos disciplinas aparentemente disímiles, como la informática y la literatura, para desarrollar una nueva propuesta de la didáctica literaria, recurriendo a la creación verbal más representativa del momento: el minicuento o minificción.

Dicho proyecto se denominó “AMBIENTE HIPERMEDIAL PARA EL DESARROLLO DE LA DIDÁCTICA LITERARIA A PARTIR DEL MINICUENTO. HIMINI” y fue aprobado por el Centro de Investigaciones de la Universidad Pedagógica Nacional para la vigencia del año 2001. A manera de síntesis se presenta a continuación los aspectos más relevantes del proceso seguido en esta investigación.

\section{Objetivos del proyecto HIMINI}

El proyecto "Ambiente Hipermedial para el desarrollo de la Didáctica literaria a partir del minicuento, HIMINI, se propuso como Objetivos fundamentales por una parte, diseñar, producir y validar un hipermedia y un portal como dispositivos de un ambiente tecnológico que permitiera el desarrollo de la didáctica literaria y facilitara el aprendizaje de los estudiantes en las formas narrativas breves y por otra, analizar el grado de desarrollo del discurso estético en la comprensión y producción de minicuentos, de un grupo de 
estudiantes de literatura del Departamento de Lenguas, a partir de la interacción con dicho ambiente.

Para alcanzar los anteriores objetivos, se diseñó el proyecto en dos fases, la primera orientada al desarrollo del ambiente hipermedial, y la segunda a la investigación propiamente dicha. Durante el año 2001 se concluyó la primera fase, que obtuvo importantes logros, todos ellos relacionados con cada una de las etapas desarrolladas para la producción del ambiente, cuyo eje temático es la comprensión y producción de minicuento. A continuación señalamos los más importantes:

1. Delimitación temática y desarrollo de contenidos

2. Elaboración de la propuesta pedagógica que funciona como substrato del ambiente hipermedial.

3. Elaboración de la propuesta de diseño.

4. Elaboración de la red conceptual

5. Diseño del Story Board

6. Búsqueda y selección de información audiovisual

7. Elaboración de librerías digitales

\section{Trasfondo teórico del proyecto}

El paradigma que sustenta este proyecto es el de la concepción bajtiniana de los estudios dialógicos (1997), (Zavala, 1991), con la cual se encuentran en franco diálogo la hermenéutica (Rícoeur, 1980) y la semiótica literarias; algunas concepciones contemporáneas de la escritura y la lectura como las de Gianni Rodari (1996) y Daniel Penac (1993), y los estudios realizados sobre la relación hipertexto/literatura, principalmente las propuestas de Landow (1995-1997) y Coovert (1992). De igual manera en él confluyen diversos planteamientos hechos desde la narrativa breve (Calvino, 1989; Polastri, 1994; Noguerol, 1995; Pérez 1997; Rojo, 1997; Rodríguez, 1996; Bustamante, 1994), y la didáctica del minicuento (Zavala, 1999); (Tomassini, 1998), todas ellas voces de la cultura y de la literatura que han contribuido a tejer la urdimbre del diálogo estético, cuyo escenario de resonancias, interlocución y réplica pretende ser el presente proyecto.

\section{Componentes del ambiente hipermedíal}

Como hemos señalado antes, en el transcurso de la primera fase el proyecto HIMINI produjo un ambiente hipermedial compuesto de un juego interactivo en formato de CD y un portal en Internet, (este último puede ser visitado en la siguiente dirección: http://himini.pedagogica.edu.co/).

Para el diseño de dicho ambiente se tuvieron en cuenta principios básicos como la lúdica y la interactividad, entendida esta última como la posibilidad que tiene el usuario de modificar, expandir y dialogar, con el ambiente. En esta medida, y luego de un exhaustivo estudio de las diferentes opciones brindadas por las tecnologías de la comunicación, se llegó a la conclusión de que los formatos más adecuados para desarrollar el HIMINI eran un juego computacional y un portal en Internet. El primero por jas posibilidades lúdica, motivacional e interactiva que puede facilitar; y el segundo, por las ventajas que brinda para la interacción maestro-alumno, alumno-alumno y alumno-conocimiento. 
Así, a cada uno de estos formatos se asignó una función y unos componentes. El portal en Internet, tiene como función por una parte, la de servir como espacio de comunicación e intercambio de ideas entre los diferentes actores del proceso de aprendizaje: profesor y estudiantes y por otra, la de posibilitar el acceso a diferentes nodos de información teórica relacionada con el minicuento así como a la antología de minicuentos, que puede ser ampliada por los estudiantes, y a la presentación del juego interactivo. Para alcanzar este propósito, el portal tiene la siguiente estructura:

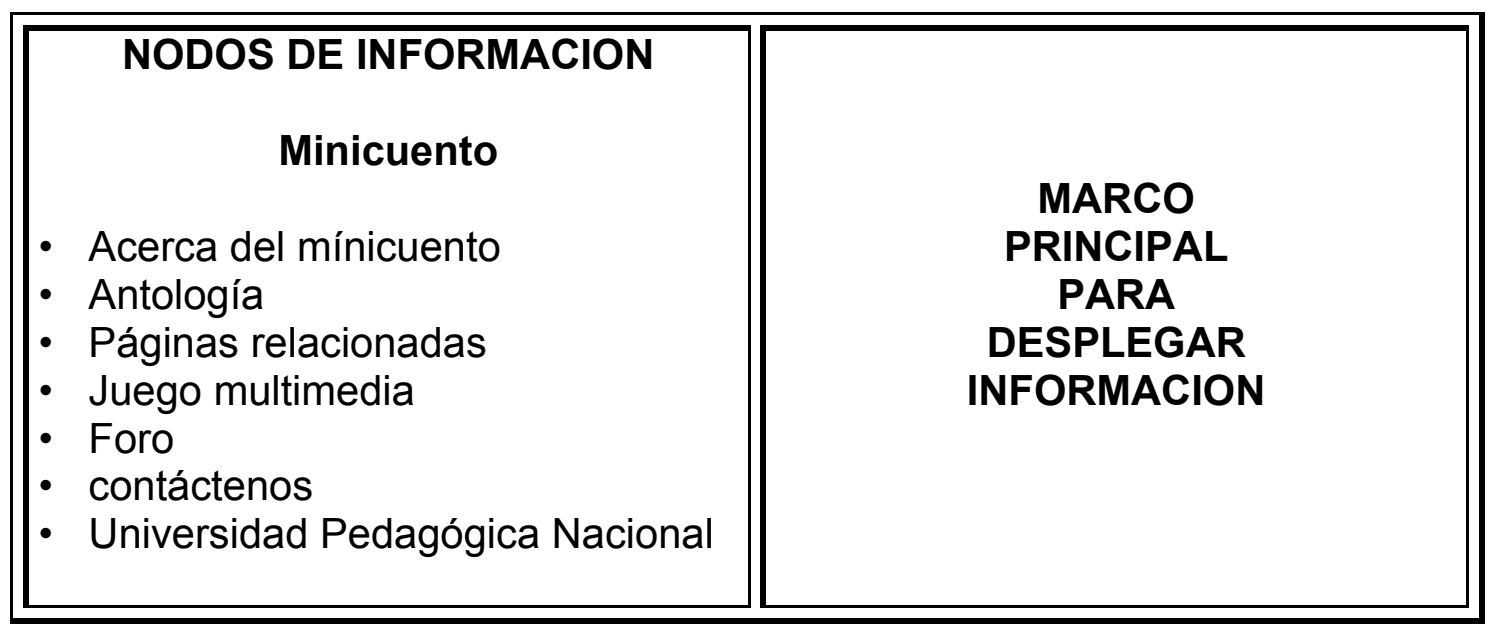

Por su parte, el juego, que hemos denominado Minificción Interactiva, es un software lúdico-didáctico diseñado a partir de estrategias pedagógico-literarias encaminadas a desarrollar y perfeccionar el discurso estético en usuarios familiarizados con la literatura y con eL uso del computador, teniendo como referente la comprensión y producción de textos breves de naturaleza narrativa.

Por medio de esta herramienta hípermedial, estudiantes y maestros pueden interactuar en torno al juego, cuyo motor creativo es la Minificción o minicuento. Su contenido se estructura en varios niveles, -expuestos más adelante- los cuales aumentan en complejidad a medida que el juego avanza y el estudiante vence los obstáculos representados por acertijos y retos. Dicho avance da cuenta de la apropiación teórica y práctica que el jugador ha hecho de las diferentes estrategias de lectura, comprensión y producción textual.

Minificción interactiva está inspirado en la metáfora que sugiere la trama narrativa de Las mi/y una noches (Casas-novas 1969; Blasco lbañez, 1978; Moreno, 1996; Rodríguez, 2001): historia tejida por múltiples historías en la que Scherezade salva su vida y la de las doncellas de su pueblo por medio de la genial estrategia de tejer historias que noche tras noche van ganando la atención del Sultán, hasta involucraría en la misma trama, haciéndolo desistir de su mortal designio. Sin embargo, en esta versión se recrea el ambiente fantástico de Las mil y una noches estilizando la trama de la historia, en la que Scherezade ya no narra cuentos clásicos durante mil y una noches sino que "vuelve a contar" esta vez, algunos mínirrelatos de fugaz duración y por poco tiempo, que es el lapso empleado por el usuario para desarrollar los desafíos que le propone el juego.

A lo anterior se suma el hecho de que la heroína cuenta con ayudantes, -quienes serán los usuarios del juego- que a manera de detectives recorren previamente lugares secretos del palacio, entre ellos la biblioteca del Sultán, y retornan a ella para informarla acerca de los secretos o claves contenidos en el tipo de relatos o microhistorias que le gusta escuchar al terrible y sanguinario gobernante, que a su vez, se transforma en un receptor con gusto estético parecido al del usuario. Así, el jugador participa de un viaje 
mágico a través de habitaciones, bibliotecas y laberintos, que no solo le llevan a descubrir los secretos de la lúdica historia sino los de su propio aprendizaje.

Al finalizar el juego, el estudiante habrá desarrollado de tal manera su percepción de las artes relacionadas con la Minificción, que él mismo podrá producir sus propios textos y así, "contribuir con la salvación de Scherezade y de su pueblo de una muerte horrible y dolorosa'.

Cada uno de los niveles que estructuran el juego tiene un propósito y una misión especificas, como se presenta a continuación:

\section{Nivel uno}

Propósito: introducir al usuario en el juego y explicarle su rol.

\section{Nivel dos}

Propósito: que el jugador se familiarice con la forma narrativa breve 138

Misión: obtener un "password" para iniciar el juego.

\section{Nivel tres}

Propósito: que el jugador identifique las características del minicuento

Misión: llegar a la sala de lectura del Sultán.

\section{Nivel cuatro}

Propósito: que el jugador comprenda algunos minicuentos.

Misión: Conocer los cuentos preferidos por el Sultán para informar a Sherezade acerca de sus características.

\section{Nivel cinco}

Propósito: que el jugador produzca minicuentos.

Misión: llegar a la fuente inspiradora para encontrar las pistas que le ayudarán a crear los minicuentos que Sherezade le contará al Sultán.

Para cumplir con la misión de cada uno de los niveles, el jugador debe resolver los acertijos y diversas situaciones (o retos) planteadas, todas las cuales están relacionadas con la compresión y producción de minicuentos. Algunas de estas actividades realizadas por el jugador son evaluadas de manera automática por el juego, pero la mayoría llegan al correo electrónico del profesor, quien adopta el rol imaginario de "Consejero de redacción de Scherezade" y luego de evaluarías decide cuándo el jugador puede obtener el password (o palabra mágica de Scherezade) para pasar al siguiente nivel.

Se debe aclarar que el "password" otorgado por el profesor es necesario únicamente cada cierto número de actividades, de manera que el jugador puede avanzaren el juego en forma autónoma, durante un número racional de eventos.

Para resolver algunos de los problemas planteados en el juego, el jugador debe buscar información contenida en el portal, por lo cual juego y portal están conectados continuamente. (cabe destacar que el portal será actualizado frecuentemente por parte del equipo de investigación). 


\section{Propuesta pedagógica}

La propuesta pedagógica que sustenta el proyecto HIMINI está basada entre otros, en los siguientes principios:

la literatura no se enseña; la didáctica de la literatura se concibe como la escenificación de un diálogo estético mediado por textos literarios, constituidos por voces que dinamizan el sentido y comprometen la réplica de los interlocutores; una concepción del juego como liberación y conocimiento profundo de la condición humana. Principios que han adquirido sustentación en la parte teórica del proyecto, pero que por razones de tiempo y espacio nos limitamos solo a mencionar aquí.

En consonancia con esos principios, se concibe el HIMINI como un ambiente de juego en el que el espacio libre dejado por la narración rápida es llenado por el usuario, ejercitando activamente la comprensión y creación de texto, a partir de lo cual deja de ser un interlocutor pasivo para convertirse en cocreador o como sostiene Gadamer, en "cojugador", del juego que le propone el HIMINI. A su vez, se considera este juego interactivo como la continuidad en el aula hipermedial del diálogo en torno a los textos literarios, que ha comenzado previamente en el aula de clase, espacio donde probablemente se han escenificado otros tantos diálogos en pos de la palabra poética que permite a los 'actores" desentrañar múltiples facetas de la condición humana y evocar una cultura de la vida en un escenario previsto para el conocimiento, el placer y la imaginación.

Esta perspectiva de lo pedagógico, sugiere una propuesta novedosa que incorporando elementos literarios y tecnológicos ofrece una alternativa para desarrollar el discurso estético de los estudiantes, de una manera dinámica, autónoma y lúdica. Así mismo, esta propuesta implica un cambio en el modelo tanto de clase (clase tradicional), como de estudiante y de maestro, pues exige asumir roles diferentes a los convencionales, puesto que el proceso de aprendizaje se da en un ambiente diferente al aula de clase, con el apoyo de recursos tecnológicos interactivos y bajo un esquema de trabajo colaborativo.

Por otra parte, la posibilidad de explorar, desde el punto de vista literario, en el estudio, comprensión y aplicación pedagógica de géneros literarios que se encuentran en auge, diferentes a los ya consolidados como el cuento y la novela, se ha enriquecido en el desarrollo de este Proyecto con el trabajo acerca del minicuento, pues, se trata de un género que cuenta con poca consideración teórica y mucho menos didáctica, aunque de gran auge y acogida en el momento. Además, la aplicación de una teoría de gran trascendencia en nuestros días como los Estudios Dialógicos ha permitido ensanchar el horizonte no solo de la indagación teórico-literaria, sino de la didáctica de la literatura.

En esta medida, se sugiere y se espera, que la interacción de los estudiantes con el HIMINI, sea una actividad imaginativa, lúdica y de conocimiento, lo cual debería hacer parte de una actividad macro, como una clase, un taller, un seminario, etc., en la que el docente de literatura asume el rol de interlocutor y dínamizador del proceso.

En consecuencia, el HIMINI será un ambiente flexible, interactivo, lúdico, de tal forma que posibilite los siguientes tipos de interactividad:

Desarrollar actividades de comprensión y producción a partir de juegos en determinado ambiente. En este ambiente se van incorporando todas las actividades posibles para comprender y producir minicuentos.

- Realizar trabajos colaborativos interactuando con el profesor y con sus compañeros. 
- Conformar un portafolio de sus producciones

- Enriquecer la antología integrada en el portal

- Participar en discusiones a través del foro del Portal

- Enriquecer sus producciones con recursos audiovisuales

- Acceder a conceptos literarios e información teórica relacionados con el minicuento.

\section{Perspectivas de la segunda fase}

En la actualidad el Proyecto HIMINI adelanta la segunda fase que comprende dos etapas: la primera, centrada en la validación y aplicación de la versión ya desarrollada del Ambiente Hipermedial HIMINI con la población sujeto 1, (estudiantes del Espacio Académico Teoría y Crítica Literarias, tercer semestre de lenguas-U.P.N.) y la segunda, en la adaptación del ambiente hipermedial y el desarrollo de esta experiencia con la población sujeto 2, (estudiantes de educación básica secundaria del Distrito Capital).

El objeto del proyecto en esta fase será indagar acerca del grado de desarrollo del discurso estético de las dos poblaciones sujeto mencionadas, a partir de su interacción con el ambiente hipermedial HIMINI.

La metodología adoptada para desarrollar la investigación es la propuesta por la poética dialógica, que tiene como fin considerar ampliamente las tres instancias fundamentales de toda creación literaria, a saber: el creador, el texto y el lector, en una perspectiva humanística.

\section{Diseño y validación de un ambiente hipermedial para el aprendizaje del inglés}

Aunque los últimos desarrollos tecnológicos han permitido que los materiales multimediales para el aprendizaje del inglés incorporen diversidad de medios de representación y transmisión del conocimiento humano, tales como el texto, las gráficas, la fotografía, la animación y el vídeo, no obstante, buena parte de esos materiales presentan tres grandes problemas, a saber:

En primer lugar, algunos de esos materiales centran su atención en el aprendizaje de ciertos componentes de la segunda lengua (L2) vistos de manera aislada. Por ejemplo, promueven el aprendizaje de vocabulario sin contexto, o de la morfología o la sintaxis sin integrar esos componentes y sin tener en cuenta ni el conocimiento fonético, ni el conocimiento pragmático explícito que el aprendiz debe aprender, paralelamente, para poder desempeñarse adecuadamente en contextos reales, con propósitos reales.

Otra falencia que presentan esos materiales multimediales es que no contienen mecanismos para determinar qué tipo de aprendiz está utilizando el software y, portante, no proporcionan ayudas adicionales para aquellos estudiantes que encuentran dificultades en su aprendizaje. En esos paquetes instruccionales, todos los aprendices que se enfrentan a las tareas propuestas reciben el mismo tratamiento y tienen acceso a las mismas ayudas. No hay manera de determinar qué tipo de problemas lingüísticos o cognitivos está afrontando el aprendiz, ni qué tipo de ayuda puede necesitar en un momento dado, al contrario de los que sucede con los llamados tutores inteligentes. En esos materiales se sigue utilizando el viejo modelo "ensayo-error" y se desperdicia toda la cantidad de investigaciones que se han realizado acerca de estilos cognitivos y estrategias de aprendizaje. 
Otra dificultad que presentan es que, aunque utilizan diversos medios que los hacen atractivos perceptualmente, éstos no logran absorber completamente al usuario, tal y como lo hacen los juegos por computador (un ejemplo de sistema desktop de realidad virtual). Puesto que dichos juegos representan mundos de 3 dimensiones dentro de los cuales los exploradores pueden viajar o moverse en cualquier dirección y pueden interactuar con el ambiente creado, a través de periféricos tales como el mouse, el teclado, el joystick o aún cascos o guantes, logran sumergir a los jugadores en la realidad virtual a la cual han ingresado y en la cual se han diseñado cuidadosamente elementos como la base de datos, la iluminación, los niveles de detalle, la aplicación de texturas, los sensores y la navegación (Cruiz, 1997). En esos mundos, los usuarios asumen un rol y escogen las herramientas que le permitirán cumplir una misión o alcanzar una meta determinada.

Con el propósito de tratar de superar estas dificultades que afectan el uso de los multimedia, formulamos el proyecto de investigación "DISEÑO Y VALIDACIÓN DE UN AMBIENTE HIPERMEDIA PARA EL APRENDIZAJE DEL INGLÉS” cofinanciado por la Universidad Pedagógica Nacional y el Centro de Investigaciones de la Universidad Pedagógica, CIUP. Entendemos por ambiente hipermedia un sistema diseñado para funcionar como parte de un equipo máquina-hombre, en el cual la tarea que debe ser solucionada por la máquina utiliza cualquier técnica que sea apropiada para el almacenamiento, captura, recuperación y despliegue de información; en la parte que debe ser solucionada por el hombre, se usa un marco hipermedia que permite comportamientos de navegación complejos con el fin de ayudarlo a manejar el complejo conocimiento almacenado (Schwabe, 2001).

En este proyecto buscamos diseñar un ambiente estructurado lógicamente, de tal manera que el estudiante de inglés logre adquirir y poner en práctica su conocimiento lingüístico (segmental y suprasegmental), pragmático (funcional) y cultural en la L2, de tal manera que le permita mejorar su comprensión auditiva y, a la vez, adquirir estrategias que le permitan llegar a convertirse en un aprendiz autónomo, que pueda continuar educándose parsi mismo, ya que como afirma McClintock, (1992) "La autonomia de una persona no es el resultado de la educación; la autonomía es la condición que la ocasiona".

Nos hemos propuesto lograr este objetivo mediante la interacción controlada del aprendiz con hablantes nativos norteamericanos e ingleses en escenarios que le proporcionen información histórica o cultural y que simulen situaciones de la vida real o de historias fantásticas. Además, que le permitan al usuario navegar de una manera no lineal. Igualmente, buscamos que capture algunos de los elementos esenciales de los juegos por computador (reglas, bonos o puntos otorgados por la ejecución correcta de una tarea, animación, entre otros), y que le permita asumir un rol determinado con el fin de llevar a cabo una gran misión, sea ésta real o fantástica.

También se creará un perfil de usuario que permita hacer un seguimiento del desempeño del aprendiz, con el fin de detectar qué grado de éxito está logrando y, de esta manera, brindarle diferentes posibilidades de ayuda. Así, aquellos que presenten dificultades en la solución de las tareas que se plantean, recibirán instrucción y/o práctica acerca del uso de estrategias de aprendizaje, más o menos avanzada, según su necesidad. De esta manera, se facilitará su proceso de aprendizaje.

Recordemos que visto desde la Ciencia Cognitiva, el proceso de aprendizaje se da mediante dos procesos: el proceso de automatización y el de re-estructuración. En las 
primeras fases del aprendizaje hay una integración gradual de habilidades mediante procesos controlados; gracias a la práctica estos procesos se vuelven automáticos. En las fases posteriores,... hay una continua re-estructuración de los componentes de una tarea, de tal manera que son coordinados, integrados y re-organizados en nuevas unidades, permitiendo que un viejo procedimiento y sus componentes sea reemplazado por uno más eficiente con componentes nuevos" (Cheng, 1985. Cit. en Mclaughlin, 1991,136).

Investigadores como Anderson, Faerch, y Kasper (en McLaughlin) establecen la distinción entre conocimiento declarativo y conocimiento procedimental. En el aprendizaje de segundas lenguas, el "qué se aprende" o conocimiento declarativo está constituido parlas reglas morfológicas, sintácticas y de uso, y por porciones de expresiones lingüísticas memorizadas. El conocimiento procedimental, en cambio, nos permite saber "cómo" utilizar ciertas estrategias y procedimientos para procesar la información lingüística que se quiere adquirir y utilizar. Se intentará que los aprendices perfeccionen sus estrategias de procesamiento de la información a nivel morfológico, sintáctico, semántico, y semio-discursivo, sus estrategias de aprendizaje, que evalúen y administren su aprendizaje y re-estructuren sus representaciones internas acerca del funcionamiento de la L2.

Teniendo en cuenta que en los últimos quince años, tanto la Linguística como la Ciencia Cognitiva han prestado atención al estudio de aquellas estrategias que diferencian a los buenos aprendices de aquellos que no lo son y que varios estudios, como los realizados por O'Malley y Chamot, en 1987, por Oxford, en 1991, y por Rost en 1996, entre otros, han tratado de enumerar, clasificar y describir estas estrategias de aprendizaje, nos estamos apoyando en ellos y en nuestra propia experiencia docente para elaborar los módulos de ayuda que ya hemos mencionado.

Es importante mencionar que en los estudios realizados por Rebecca Oxford se presentan 12 rasgos de dichas estrategias, los cuales hacen posible el desarrollo de una competencia comunicativa en el aprendiz. Las estrategias:

- Contribuyen a desarrollar aspectos particulares de la competencia comunicativa, a saber: las competencias gramatical, socio-lingüística, discursiva y la estratégica.

- Les permiten a los estudiantes ser más auto-dirigidos, es decir, les ayudan a dirigir mejor sus esfuerzos tendientes a lograr el aprendizaje).

- Expanden el rol del profesor.

- Están orientadas hacia la resolución de problemas.

- Son acciones específicas tomadas por el aprendiz.

- Involucran otros aspectos del aprendiz, no sólo lo cognitivo.

- Apoyan el aprendizaje directa e indirectamente.

- A menudo son conscientes.

- Pueden ser enseñadas.

- Son flexibles.

- Son afectadas por una variedad de factores (estadio de aprendizaje, requerimientos de la tarea, edad, sexo, nacionalidad, estilo de aprendizaje general, personalidad, motivación y el propósito que se tiene para aprender una lengua

De acuerdo con O'Malley y Chamot (1996) existen 7 tipos de estrategias metacognitivas que permiten al aprendiz planear, administrar y evaluar su desempeño al ejecutar una tarea específica, a saber: 
Planificación

Atención dirigida

Atención selectiva

Auto-administración

Auto-monitoreo

Monitoreo de la

Comprensión

Monitoreo de la producción

Monitoreo auditivo

Monitoreo visual

Monitoreo del estilo

Por su parte Rost (1996), menciona algunas de las estrategias que un oyente debe utilizar para darle sentido a lo que escucha. Entre esas estrategias tenemos la diferentes formas que se deben utilizar para reconocer los límites de la palabras y para editar el discurso que se escucha, de tal manera que se tengan en cuenta dichos límites. También es importante que se aprenda a reconocer los grupos rítmicas, y el uso de la entonación descendente, plana o ascendente, sea para distinguir entre información "dada" (la que es compartida por el hablante y el oyente) o información 'nueva', o sea para saber cuando interrumpir al hablante y tomar la palabra en una conversación (Beattie en Rost, 1996).

En nuestra investigación buscamos determinar si el uso del ambiente hipermedial anteriormente descrito produce cambios significativos en la forma en que los participantes se enfrentan a tareas de tipo auditivo e interactivo en la L2. Ese estudio se llevará a cabo mediante el análisis pretest-postest, y el análisis de protocolos (Question-asking protocol), pero utilizando preguntas directas que los usuarios deben responder a medida que utilizan el prototipo (Hom, 1998).

Evaluación de la habilidad Evaluación de la estrategia Evaluación del repertorio lingüístico

\section{BIBLIOGRAFÍA}

BAJTIN, Mijail. 1997. 'La palabra en la vida y la palabra en la poesía. Hacia una poética sociológica”. En Hacia una filosofía del acto ético. Anthropos. Barcelona.

BROWN, Edwar; Chignell, Mark. 1993. Learning by linking: pedagogical enviranments for hypermedia authoring. En journal of computer in higher education. Vol. 5 \#1.

BUSTAMANTE, Guillermo y KREMEH, Harold. 1994. Antología del cuento corto colombiano. Universidad del Valle. Cali.

CALVINO, Italo. 1989. Seis propuestas para el próximo milenio. Siruela. Madrid

COOVERT, Robert. 1992. Finding your way in hipertext. En New York Times _Book Review.

CRUIZ, Hernando.1997. Diseñando aplicaciones de realidad virtual para el desktop. Revista Interamericana de Nuevas Tecnologías de la Información Vol $\underline{3}$ Nol y 2.

HOM, James. 1996. The Usability Method Toolbox. 
LANDOW, George. 1995. Hipertexto: La convergencia entre la teoría crítica contemporánea y la tecnología. Paidós. Barcelona.

1997. Teoría del hipertexto. Paidós. Barcelona.

LAS MIL Y UNA NOCHES. (Antología, 2001). Compilación y prólogo a cargo de Margarita Rodríguez Acero. Ediciones Longseller, 2001.

(1969). Edición escolar preparada por Luis Casasnovas Márques. Editorial Everest. León-España.

1996. Selección de Martin Moreno. Editorial Panamericana. Bogotá.

EL LIBRO DE LAS MIL Y UNA NOCHES (1978). Versión de Vicente Blasco Ibañez, ilustraciones de Juan Marigot. Circulo de Lectores. Barcelona.

MCLAUGHIN. 1991 Theories of Second Language Learning. Edward Arnold London.

MCCLINTOCK, Robbie. 1992. Power and Pedagogy: Transforming Education through Information Technology http://www.ilt.columbia.edu/publications/index.html

NOGUEROL JIMÉNEZ, Francisca. 1995. La trampa en la sonrisa. Sátira en a narrativa de Augusto Monterroso. Universidad de Sevilla. Sevilla.

O’MALLEY, Michael; Chamot, Anca. 1996. LearnIng Strategies in Second Language Acquisition. Cambridge Applied Linguistics. Cambridge.

OXFORD, Rebecca. 1990. Languaqe learning Strategies: What every teacher should know. Heinle \& Heinle publishers. Boston.

PENNAC, Daniel. 1992. Como una novela. Editorial Norma. Bogotá

PÉREZ BELTRAN, Ángela María. 1997. Cuento y minicuento. Página Maestra Editores. Bogotá.

POLLASTRI, Laura. 1994. 'Una escritura de lo intersticial: las formas breves en la narrativa hispanoamericana contemporánea". En El puente de las palabras. Homenaje a David Lagmanovich. OEA INTERAMER. Washington.

RICOEUR, Paul. 1980. La metáfora viva. Ediciones Cristiandad. Madrid

RODARI, Gianni. 1996. Gramática de la fantasia. Ediciones del Bronce. Barcelona

RODRIGUEZ ROMERO. Nana. 1996 Elementos para una teoría de minicuento. Colibri Ediciones. Tunja

ROJO, Violeta. 1997. Breve Manual para reconocer minicuentos. UAM. Mexico.

ROST. Michae!. 1992. Listening in Lanquage Learning. Longman, London.

SCHWABE. Daniem, ROSSI Gustavo. 2001. The Object Oriented Hypermedia Design Model. www.di.inf.puc rio.br/—schawbe 
TOMASSIN!, Graciela y MARIS COLOMBO Stella. 1998 Comprensión lectora y producción textual. Minificción híspanoamericana. Fundacion Ross. Rosario

ZAVALA. Lauro. (Compilador). 1999. Lecturas simultaneas: la enseñanza de lengua y literatura con especia! atención al cuento ultracorto. Universidad Autónoma Metropolitana. México.

ZAVALA, Iris M. La posmodernidad y Mtjati Bajtin. Una Poética Dialógica Madrid Espasa Calpe, 1991. 